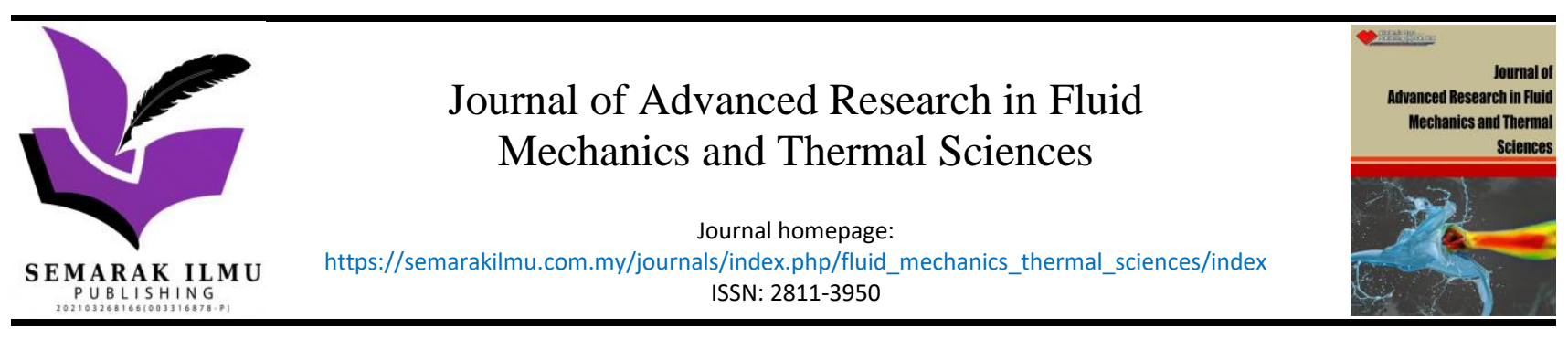

\title{
Nonlinear Convective Flow of Williamson Fluid Over a Slendering Stretching Sheet with Melting Heat Transfer
}

\author{
Mocherla Gayatri ${ }^{1, *}$, Konda Jayarami Reddy ${ }^{2}$, Bommanna Lavanya ${ }^{3}$ \\ Department of Mathematics, Research Scholar, Koneru Lakshmaiah Education Foundation, Vaddeswaram, Andhra Pradesh, 522302, India \\ Department of Mathematics, Koneru Lakshmaiah Education Foundation, Vaddeswaram, Andhra Pradesh, 522302, India \\ Department of Mathematics, Manipal Institute of Technology, Manipal Academy of Higher education, Manipal, Karnataka, 576104, India
}

\section{ARTICLE INFO}

\section{Article history:}

Received 29 October 2021

Received in revised form 20 December 2021

Accepted 26 December 2021

Available online 16 January 2022

\section{Keywords:}

Grashof number; Williamson fluid; melting surface; non-uniform thickness; shooting technique; chemical reaction; thermal radiation

\section{ABSTRACT}

The main concern of this paper is to analyze the nonlinearity of the Grashof numbers (thermal and diffusion) on the Williamson fluid flow across a stretching sheet with nonuniform thickness with the consideration of melting heat transfer. The modelled equations are non-dimensionalized with the utilization of similarity transformations. We used shooting technique based on Runge-Kutta $4^{\text {th }}$ order method to unriddle the ensuing equations. Influence of key parameters on the common profiles i.e., velocity, temperature and concentration are deliberated with the support of graphs. And also, we examined friction factor, local Sherwood and Nusselt numbers against the same parameters and displayed the results in tabular form. It is seen that Weissenberg number and wall thickness parameters rise the magnitude of friction factor. It is additionally qualified to specify that the temperature and concentration profiles looking high in the absence of melting parameter contrasted with the presence of melting parameter.

\section{Introduction}

During the most recent couple of years, analysts appear to be keen on investigating liquefying heat exchange because of across the board utilizations of freezing and melting in magma solidification, potential heat thermal energy storage, casting of an assembling procedure, welding process, and crystal growth and so on. So extensive measure of test and hypothetical research has been completed to decide the part of natural convection in the dynamics of heat transfer attached to solidification or melting impact. With a specific end goal to control the major parameters, for example, the speed of manufacture, rate of deformities and additionally the impact on the final properties of items and the likelihood of harm of the contact surface between the wall and phase change material, the forecast of temperature appropriation, solidification or melting rate is critical in some cutting-edge advancements. Initially, Epstein and Cho [1] introduced an exact closed- form solution for the case of porous media. They noticed that the influence of the Prandtl number on the

\footnotetext{
* Corresponding author

Email address: gayatri.mocherla@gmail.com
}

https://doi.org/10.37934/arfmts.91.1.1834 
normalized Nusselt number disappears as it becomes large. Bachok et al., [2] used to Runge-KuttaFehlberg method to solve the equations which are raised in the problem of two-dimensional stagnation point flow across a horizontal stretching/shrinking sheet in the presence of melting heat transfer. They found that melting parameter reduces the skin friction coefficient. Ishak et al., [3] examined the melting phenomena on the forced convective flow across a moving surface. They observed that melting strength lessen the rate of heat transfer at the solid-fluid interface. By considering viscous dissipation, the characteristics of melting heat transfer on the stagnation-point flow of Jefferey fluid were analysed by Mustafa et al., [4] and concluded that the influence of melting parameter and the Prandtl number is quite opposite on temperature and velocity profiles. Hayat et al., [5-7] investigated stagnation-point flows of different fluids across a stretching surface by viewing several parameters including Soret and Dufour along with melting heat transfer. Some of their findings are (a) Prandtl enhances the rate of heat transfer (b) melting parameter lessen the magnitude of local Sherwood number (c) mixed convection parameter improves the velocity profile. Das et al., [8] used MAPLE 17 to obtain the numerical results for the problem of Jeffrey fluid flow across a stretching surface with the consideration of some parameters including surface slip and melting heat transfer and found that Jeffrey fluid enhances the temperature compare to Newtonian fluid. By considering stretching sheet, Mabood and Antonio and Gireesha et al., [9-10] studied the flow of nanofluid in the presence of various parameters including melting and thermophoresis. The part of their results and discussion contains many points including Prandtl number enhances the rate of mass transfer and thermophoresis shows the same kind of behaviour on the Nusselt number. Mabood and Das, Krishnamurthy et al [11-12] reported that the rate of mass transfer is high at the wall in case of no-slip condition and non-linear stretching parameter enhances the friction factor in the problem of two dimensional MHD flow of a fluid across a stretching sheet by viewing chemical reaction and melting parameter. Animasaun [13] used shooting technique based on fourth order R$K$ Gill method to solve equations (transformed) which are raised in the problem of mixed convective stagnation micropolar fluid flow across a stretching sheet by considering variable thermal conductivity along with melting and other parameters and noticed that variable thermal conductivity lessen the local Nusselt number. Hashim [14] and Sheikholeslam et al., [15-16] studied the characteristics melting heat transfer of various fluids across various channels including stretching plate. They witnessed that temperature improved with bigger Prandtl number and melting parameter raises the nanofluid concentration.

Thermal radiation is developed by the movement of charged particles in subject. Generally, their movement is in a shape of waves (electromagnetic) and look like as if they were from room heater and electric burner. With regards to space innovation and in the procedures including high temperatures, the impact of radiation assumes an indispensable job. Initially, by employing Rosseland approximation to alter the energy equation, Viskanta and Grosh [17] studied the influence of thermal radiation on an incompressible flow and concluded that radiation parameter enhances the thickness of the thermal boundary layer. Gupta and Gupta [18] elucidated the influence of radiation on the mixed convective fluid flow within an open-ended vertical channel and observed that the radiation applies a balancing out impact on the flow which is reliable with the way that radiation will in general decrease the impact of regular convection. B.Lavanya [18] studied MHD Rotating Flow Through a Porous Medium with Heat and Mass Transfer. The study of the interaction of thermal radiation with the natural convection was conducted by Ali [19] in the domain of semiinfinite flat plate. Hossain and Takhar [20] noticed that the Prandtl number lessens the local shear stress in the three convective modes (free, forced and mixed) in their work on the boundary layer flow across a vertical flat plate in the presence of thermal radiation. Israel-Cookey et al., [21] and Raptis et al., [22] explained the MHD flow across different plates by considering several parameters 
including Grashoff number along with thermal radiation. Darcy parameter lessens the velocity and radiation parameter enhances the normalized similarity temperature are some of their observations. With the aid of Runge-Kutta fourth order based shooting method, Cortell [23] resolved the transmuted equations for the fluid flow across a flat sheet and mentioned that wall thickness parameter lessens the velocity. By considering vertical porous plate, Mohamed and Abo-Dahab [24] and Mukhopadhyay [25] demonstrated different flows by viewing various parameters including unsteadiness, thermal radiation and chemical reaction. Some of their results are unsteadiness parameter enhances the heat transfer rate and Schmidt number lessens the velocity. M.Nagasasikala et.al [26] studied Heat and Mass Transfer of a MHD Flow of a Nanofluid Through a Porous Medium in an Annular, Circular Region with Outer Cylinder Maintained at Constant Heat Flux. Bhattacharyya and Layek [27] provided dual solutions in the analysis of stagnation point flow across a shrinking sheet with thermal radiation. Later several researchers [29-35] contributed their work of various fluid flows in the domain of different surfaces in the presence of thermal radiation along with some other parameters.

The present work is partly taken from Hayat et al., [33] and we have extended it by considering nonlinear convection parameter together with chemical reaction and thermal radiation. Transformed the governing equations as ordinary differential equations with the aid of similarity variables and then the equations resolved by using Runge-Kutta fourth order method based shooting technique. We have provided solutions in two cases i.e., in the presence and absence of melting heat transfer and used plots to display them. We have also analyzed the friction factor, heat and mass transfer rates in tabular form against the same parameters for which we have graphs.

\section{Mathematical Analysis}

A two-dimensional Williamson fluid flow across a stretching sheet with non-uniform thickness in the manifestation of thermal radiation and chemical reaction is examined. An inhomogeneous magnetic field of strength $B_{0}$ is carried out normal to the sheet. We have analyzed the flow with melting heat transfer. Furthermore, we assume that the stretching velocity is $U_{w}(x)=A(x+q)^{m} f^{\prime}$ in the $\mathrm{x}$-direction, $y=\varepsilon(x+q)^{1-m / 2}$ (where $\varepsilon$ is small so that the surface is sufficiently thin) and considered Reynolds's number as much as less to push away the stimulated magnetic field.

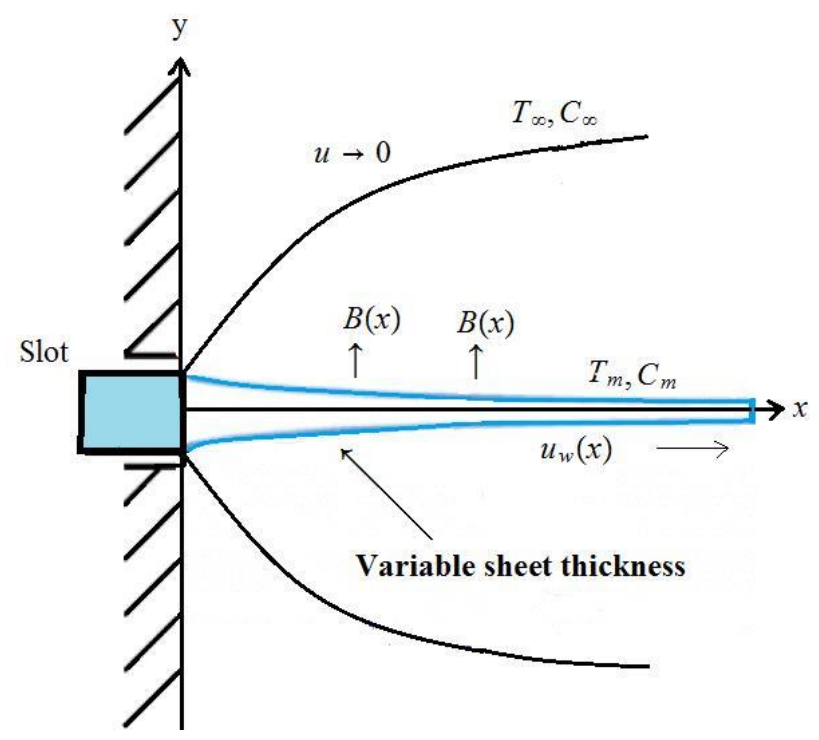

Fig. 1. Physical model of the problem 
In 1929, Williamson proposed a model to examine the pseudoplastic fluid flow. Not like the other models, this model considers the minimum and maximum viscosities of the fluid which are needed for pseudoplastic fluids. The underlying equations of the Williamson fluid model are established as [34]:

$S=-p I+\tau$

$\tau=\left[\mu_{\infty}+\frac{\left(\mu_{0}-\mu_{\infty}\right)}{1-\Gamma \dot{\gamma}}\right] A_{1}$

Where $p$ is the pressure, $I$ is the identity vector, $\tau$ is the extra stress tensor, $\mu_{0}$ and $\mu_{\infty}$ are the limiting viscosities at zero and at infinite shear rate, $\Gamma \succ 0$ is the time constant, $A_{1}$ is the first Rivlinrickson tensor.

$\dot{\gamma}$ is defined as follows:

$\dot{\gamma}=\sqrt{\frac{1}{2} \pi}$

Where $\pi=\operatorname{trace}\left(A_{1}^{2}\right)$,

$\dot{\gamma}=\left[\left(\frac{\partial u}{\partial x}\right)^{2}+\frac{1}{2}\left(\frac{\partial u}{\partial y}+\frac{\partial v}{\partial x}\right)^{2}+\left(\frac{\partial v}{\partial y}\right)^{2}\right]^{1 / 2}$

If $\mu_{\infty} \succ 0$ and $\Gamma \dot{\gamma} \prec 1$, then (1.2) can be written as

$\tau=\left[\frac{\mu_{0}}{1-\Gamma \dot{\gamma}}\right] A_{1}$

With the help of binomial expansion, (1.5) can be expressed as

$\tau=\mu_{0}[1+\Gamma \dot{\gamma}] A_{1}$

With these assumptions, the governing equations [33] are

$\frac{\partial u}{\partial x}+\frac{\partial v}{\partial y}=0$ 


$$
\begin{aligned}
& \left.u \frac{\partial u}{\partial x}+v \frac{\partial u}{\partial y}=v \frac{\partial^{2} u}{\partial y^{2}}+\sqrt{2} v \Gamma\left(\frac{\partial u}{\partial y}\right)\left(\frac{\partial^{2} u}{\partial y^{2}}\right)+g \beta_{T}\left(T-T_{m}\right)+g \beta_{1 T}\left(T-T_{m}\right)^{2}\right) \\
& +g \beta_{C}\left(C-C_{m}\right)+g \beta_{1 C}\left(C-C_{m}\right)^{2}-\frac{\sigma B^{2}(x)}{\rho} u \\
& u \frac{\partial T}{\partial x}+v \frac{\partial T}{\partial y}=\frac{k}{\rho c_{p}} \frac{\partial^{2} T}{\partial y^{2}}+\frac{\mu}{\rho c_{p}}\left(\left(T_{\infty}-T_{m}\right)\left(\frac{\partial u}{\partial y}\right)^{2}\right)+\frac{1}{\rho c_{p}} \frac{16 \sigma^{*} T_{\infty}^{3}}{3 k^{*}} \frac{\partial^{2} T}{\partial y^{2}} \\
& u \frac{\partial C}{\partial x}+v \frac{\partial C}{\partial y}=D_{m} \frac{\partial^{2} C}{\partial y^{2}}-k_{0}\left(C-C_{m}\right)
\end{aligned}
$$

And the right boundary conditions which are suitable to the present problem are mentioned as

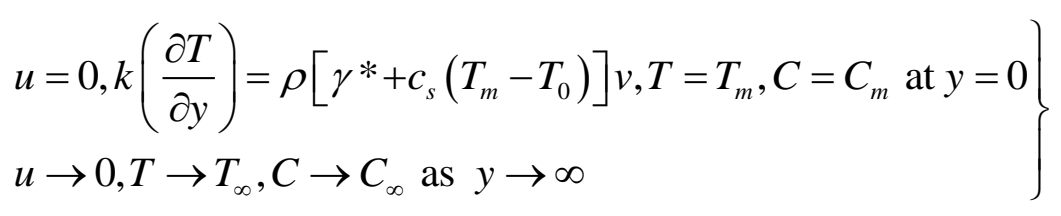

where $u, v$ are the velocity elements in parallel to $\mathrm{x}$ and $\mathrm{y}$ directions respectively, $v$ is the kinematic viscosity, $\Gamma$ is the dimensional Williamson parameter, $\mathrm{g}$ is the gravity field, $\beta_{T}$ is the volumetric coefficient of thermal expansion, $T_{m}$ is the melting surface temperature, $\beta_{C}$ is the volumetric coefficient of diffusion expansion, $\sigma$ is the electrical conductivity, $\rho$ is the density of the fluid, $B(x)$ is the dimensional magnetic field parameter, $k$ is the thermal conductivity, $C_{p}$ is the specific heat capacitance, $\mu$ is the dynamic viscosity, $\sigma *$ is the Stefan-Boltzmann constant, $k *$ is mean absorption coefficient, $C$ is the dimensional concentration parameter, $D_{m}$ is the molecular diffusivity, $k_{0}$ is the dimensional chemical reaction parameter, $\gamma^{*}$ is the latent heat, $C_{s}$ is the heat capacity of the solid, $T_{0}$ is the temperature of the solid.

The needed transformations to decrypt the regulating equations as a set of non-linear ordinary differential equations are given below:

$$
\begin{aligned}
& \zeta=y \sqrt{\left(\frac{m+1}{2}\right) \frac{A}{v}}(x+q)^{m-1 / 2},
\end{aligned}
$$

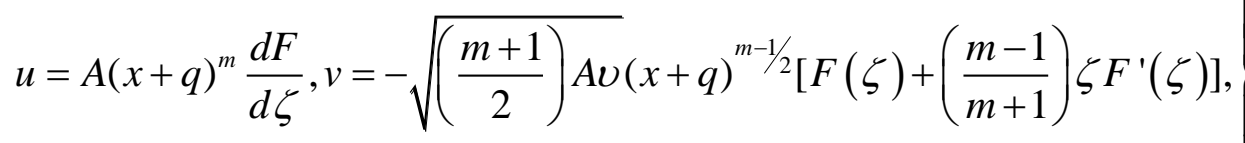

$$
\begin{aligned}
& \Theta(\zeta)=\frac{T-T_{m}}{T_{\infty}-T_{m}}, \Phi(\zeta)=\frac{C-C_{m}}{C_{\infty}-C_{m}}
\end{aligned}
$$

By using Eq. (12), Eq. (8) - Eq. (10) transmuted as the following equations: 


$$
\begin{aligned}
& {\left[1+\Lambda \frac{d^{2} F}{d \zeta^{2}}\right] \frac{d^{3} F}{d^{3} \zeta}+f \frac{d^{2} F}{d \zeta^{2}}-\frac{2 m}{m+1}\left(\frac{d F}{d \zeta}\right)^{2}+\frac{2}{m+1}\left[\operatorname{Gr} \Theta+G r 1\left(\Theta^{2}\right)+G c \Phi+G c 1(\Phi)^{2}-M \frac{d F}{d \zeta}\right]=0} \\
& \left(1+\frac{4}{3 R}\right) \frac{d^{2} \Theta}{d \zeta^{2}}+\operatorname{Pr}\left[f \frac{d \Theta}{d \zeta}+E c\left(\frac{d^{2} F}{d \zeta^{2}}\right)^{2}\right]=0 \\
& \frac{d^{2} \Phi}{d \zeta^{2}}+S c\left(f \frac{d \Phi}{d \zeta}-\frac{2}{m+1} \operatorname{Kr} \Phi\right)=0
\end{aligned}
$$

and the boundary conditions (11) changed as

$$
\left.\begin{array}{l}
\left.\frac{d F}{d \zeta}\right|_{\zeta=0}=0,\left.M e \frac{d \Theta}{d \zeta}\right|_{\zeta=0}+\operatorname{Pr}\left[F(0)+\frac{m-1}{m+1} \delta\right]=0, \Theta(0)=0, \Phi(0)=0, \\
\left.\frac{d F}{d \zeta}\right|_{\zeta=\infty}=0, \Theta(\infty)=1, \Phi(\infty)=1
\end{array}\right\}
$$

where $\delta=\varepsilon \sqrt{\frac{m+1}{2} \frac{A}{v}}$ is the wall thickness parameter. Eq. (13) - (15) can be transformed into the system of dimensionless expressions by utilizing $F(\zeta)=f(\zeta-\delta)=f(\varsigma)$,

$\Theta(\zeta)=\theta(\zeta-\delta)=\theta(\varsigma), \Phi(\zeta)=\phi(\zeta-\delta)=\phi(\varsigma)$.

The resultant equations are

$$
\begin{aligned}
& {\left[1+\Lambda \frac{d^{2} f}{d \varsigma^{2}}\right] \frac{d^{3} f}{d^{3} \varsigma}+f \frac{d^{2} f}{d \varsigma^{2}}-\frac{2 m}{m+1}\left(\frac{d f}{d \varsigma}\right)^{2}+\frac{2}{m+1}\left[G r \theta+G r_{1}\left(\theta^{2}\right)+G c \phi+G c_{1}(\phi)^{2}-M \frac{d f}{d \varsigma}\right]=0} \\
& \left(1+\frac{4}{3 R}\right) \frac{d^{2} \theta}{d \varsigma^{2}}+\operatorname{Pr}\left[f \frac{d \theta}{d \varsigma}+E c\left(\frac{d^{2} f}{d \varsigma^{2}}\right)^{2}\right]=0 \\
& \frac{d^{2} \phi}{d \varsigma^{2}}+\operatorname{Sc}\left(f \frac{d \phi}{d \varsigma}-\frac{2}{m+1} K r \phi\right)=0
\end{aligned}
$$

And the resultant boundary conditions are

$$
\left.\begin{array}{l}
\left.\frac{d f}{d \varsigma}\right|_{\varsigma=0}=0,\left.M e \frac{d \theta}{d \varsigma}\right|_{\varsigma=0}+\operatorname{Pr}\left[f(0)+\frac{m-1}{m+1} \delta\right]=0, \theta(0)=0, \phi(0)=0, \\
\left.\frac{d f}{d \varsigma}\right|_{\varsigma=\infty}=0, \theta(\infty)=1, \phi(\infty)=1
\end{array}\right\}
$$


Here Weissenberg parameter $\Lambda$, thermal Grashof number $G r$, nonlinear thermal Grashof number $G r_{1}$, diffusion Grashof number $G c$, nonlinear diffusion Grashof number $G c_{1}$, Magnetic field parameter $M$, radiation parameter $R$, Prandtl number Pr, Eckert number $E c$, Schmidt number $S c$, chemical reaction parameter $K r$, melting heat transfer parameter $M e$ are defined as follows:

$$
\left.\begin{array}{l}
\Lambda=\Gamma \sqrt{(m+1) A \frac{(x+q)^{3 m-1}}{v}}, G r=\frac{g \beta_{T}\left(T_{\infty}-T_{m}\right)}{A^{2}(x+q)^{2 m-1}}, G r_{1}=\frac{g \beta_{1 T}\left(T_{\infty}-T_{m}\right)^{2}}{A^{2}(x+q)^{2 m-1}}, \\
G c=\frac{g \beta_{C}\left(C_{\infty}-C_{m}\right)}{A^{2}(x+q)^{2 m-1}}, G c_{1}=\frac{g \beta_{1 C}\left(C_{\infty}-C_{m}\right)^{2}}{A^{2}(x+q)^{2 m-1}}, M=\frac{\sigma B_{0}{ }^{2}}{A \rho}, R=\frac{k k^{*}}{4 \sigma^{*} T_{\infty}^{3}}, \\
\operatorname{Pr}=\frac{\mu c_{p}}{k}, E c=\frac{A^{2}(x+q)^{\frac{5 m-1}{2}}}{c_{p}}, S c=\frac{v}{D_{m}}, K r=\frac{k_{0}}{A(x+q)^{m-1}}, M e=\frac{c_{p}\left(T_{\infty}-T_{m}\right)}{\left[\gamma^{*}+c_{s}\left(T_{m}-T_{0}\right)\right]}
\end{array}\right\}
$$

The usual non-dimensionalization parameters of interest, friction factor $C_{f}$, the local Nusselt and Sherwood numbers $\mathrm{Nu}$ and $\mathrm{Sh}$ are defined as the following:

$$
\left.\begin{array}{l}
C_{f}=\left.\left(\operatorname{Re}_{x}\right)^{-0.5} \sqrt{\frac{m+1}{2}} \frac{d^{2} f}{d \varsigma^{2}}\right|_{\varsigma=0}, N u=-\left.\left(\operatorname{Re}_{x}\right)^{0.5} \sqrt{\frac{m+1}{2}} \frac{d \theta}{d \varsigma}\right|_{\varsigma=0} \\
\operatorname{Sh}=-\left.\left(\operatorname{Re}_{x}\right)^{0.5} \sqrt{\frac{m+1}{2}} \frac{d \phi}{d \varsigma}\right|_{\varsigma=0}
\end{array}\right\}
$$

Where $\operatorname{Re}_{x}$ is defined as $\operatorname{Re}_{x}=\frac{U_{w}(x)}{v}(x+q)$

\section{Numerical Procedure}

Numerical solutions of profoundly nonlinear customary differential Eq. (17) - Eq. (19) related with the boundary conditions (20) are determined by shooting technique dependent on Runge-Kutta fourth order method. The fundamental step of computational scheme incorporates the accompanying focuses.

Reduce Eq. (17) - Eq. (19) into system of $1^{\text {st }}$ order equations by utilizing reasonable substitution characterized as follows: 


$$
\left.\begin{array}{l}
f_{1}=f, \\
f_{2}=\frac{d f_{1}}{d \varsigma}=\frac{d f}{d \varsigma}, \\
f_{3}=\frac{d f_{2}}{d \varsigma}=\frac{d^{2} f}{d \varsigma^{2}}, \\
f_{4}=\theta(\varsigma), \\
f_{5}=\frac{d f_{4}}{d \varsigma}=\frac{d \theta}{d \varsigma}, \\
f_{6}=\phi(\varsigma), \\
f_{7}=\frac{d f_{6}}{d \varsigma}=\frac{d \phi}{d \varsigma}
\end{array}\right\}
$$

By using this, we can write

$$
\left.\begin{array}{l}
\frac{d f_{1}}{d \varsigma}=f_{2}, \\
\frac{d f_{2}}{d \varsigma}=f_{3}, \\
\frac{d f_{3}}{d \varsigma}=\left(\frac{1}{1+\Lambda f_{3}}\right)\left(\frac{2 m}{m+1} f_{2}^{2}-f_{1} f_{3}-\frac{2}{m+1}\left(G r f_{4}+G r_{1} f_{4}^{2}+G c f_{6}+G c_{1} f_{6}^{2}-M f_{2}\right)\right) \\
\frac{d f_{4}}{d \varsigma}=f_{5}, \\
\frac{d f_{5}}{d \varsigma}=-\frac{\operatorname{Pr}}{\left(1+\frac{4}{3} R\right)}\left[f_{1} f_{5}+E c f_{3}^{2}\right] \\
\frac{d f_{6}}{d \varsigma}=f_{7}, \\
\frac{d f_{7}}{d \varsigma}=-S c\left(f_{1} f_{5}-\frac{2}{m+1} K r f_{6}\right)
\end{array}\right\}
$$

We can use the same procedure for the boundary conditions mentioned in (20). After the conversion, we can apply the bvp5c MATLAB package to get the appropriate results.

\section{Results and Graphs}

The set of ordinary differential Eq. (17) - Eq. (19) with respect to the boundary conditions (20) are settled numerically by applying shooting technique based on Runge-Kutta fourth order method. We exhibited the impact of non-dimensional parameters, for example $G r_{1}, G c_{1}, R$, on velocity, concentration and temperature using plots and the impact of the same parameters on friction factor, local Nusselt number and Sherwood numbers using tables. In the present investigation, we examine the results in two cases i.e., presence and absence of melting surface and we have obtained solutions by keeping $M e=1.1$ and $M e=0$. 
Figure 2-4 show the effect of the magnetic field on the common profiles (velocity, temperature, concentration) for both $M e=1.1$ and $M e=0$. From Figure 2-4, it is evident that the strength of magnetic field is to diminish the velocity, temperature and concentration fields. This reduction can be attributed to the fact that the magnetic field provides a resisting type force known as the Lorentz force. This force tends to lessen the motion of fluid and as a consequence, the velocity depreciates. The temperature and concentration fields are high in $M e=0$ when compared to $M e=1.1$. Figure 5 and 6 represent the plots of temperature and velocity with variation in $R$. The temperature increases rapidly throughout the boundary layer owing to the release of energy in thermal boundary layer for increasing values of the thermal radiation. In physically as rising values of thermal radiation encourages the generation of thermal heat energy in the flow, due to this boosting phenomena in velocity and temperature. The impact of Eckert number that it improves the thermal boundary layer, lessens the concentration and shows mixed performance in velocity is perceived in Figure 7-9. Since as improving Eckert number the dissipation among the particles is increase this helps to improve the boundary layer near the surface velocity and surface temperature. But due to unsteadiness domination, we saw decrement in diffusion boundary layer.

Figure 10-12 are plotted to demonstrate the influence of the chemical reaction on the temperature, concentration and velocity. It displays that the concentration, velocity and temperature fields are reduced with incrementing values of chemical reaction. The concentration profiles are high in the non-melting surface condition when compared with melting surface condition. Figure 13-15 observed the temperature and concentration fields are decreased and mixed performance in velocity fields respectively with improving values of Weissenberg number. As we expected, when we incorporate the non-Newtonian fluid, it improves the pressure on the flow. But in this study, due to domination of nonlinear convection in the flow, initially we saw depreciation after reaches certain limit enhancement in the flow. In Figure 16-18 shows the influence of wall thickness on velocity, temperature and concentration field in both cases. As rising of wall thickness improves the thickness of the boundary layer, wall thickness helps to depreciate the temperature and concentration boundary, whereas it shows mixed behaviour on velocity. The impact of nonlinear thermal convection on velocity, temperature and concentration fields is depicted in Figure 19 - 21. Note that improving $G r_{1}$ shows encouragement in all the profiles ( $f^{\prime}(\varsigma), \theta(\varsigma) \phi(\varsigma)$ ) of the flow. In the presence of melting surface condition, the momentum boundary layer is initially and at the end of the surface is high compared to the absence of melting surface condition. Figure 22-24 depicts the $f^{\prime}(\varsigma), \theta(\varsigma) \phi(\varsigma)$ profiles against the nonlinear diffusion convection parameter. We observed that the nonlinear diffusion convection parameter improves concentration in the flow, and furthermore this helps to boost the momentum, diffusion and concentration boundary layers for both the cases.

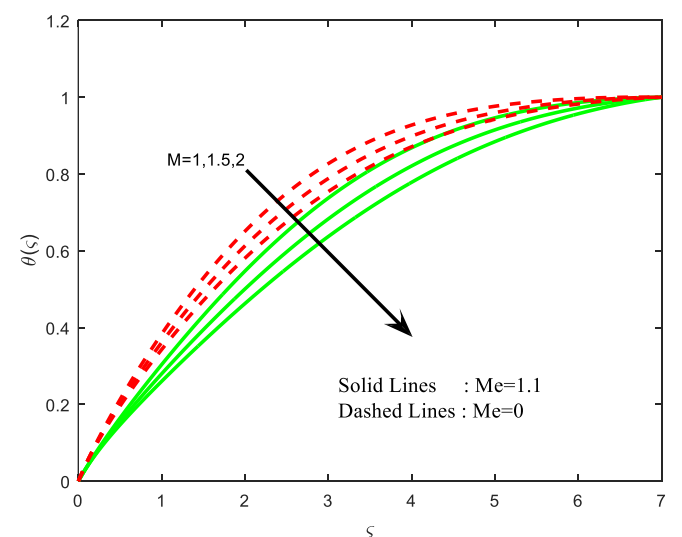

Fig. 2. Impact of $M$ on $\theta(\varsigma)$

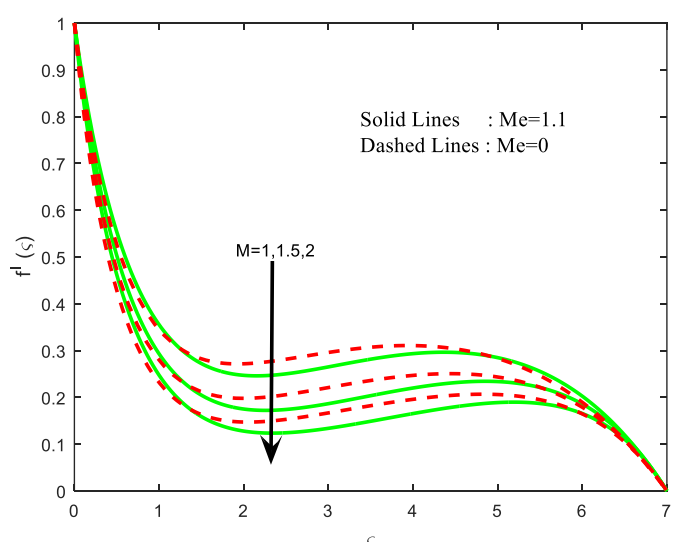

Fig. 3. Impact of $M$ on $f^{\prime}(\varsigma)$ 


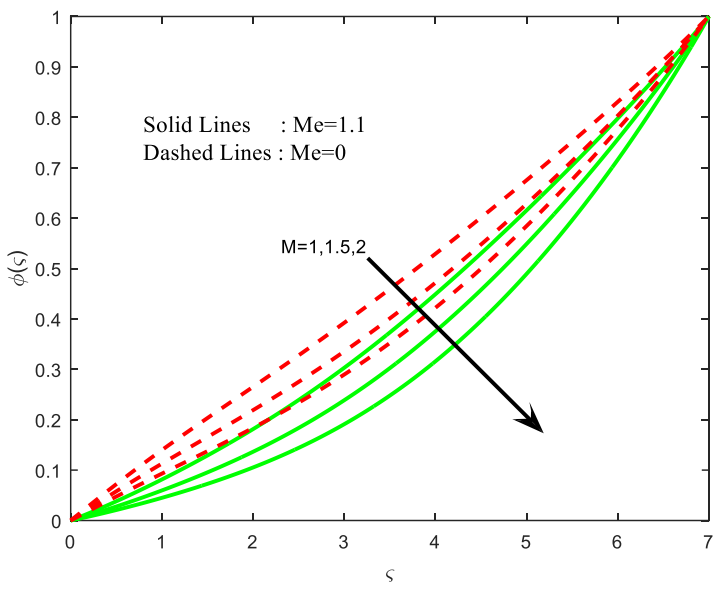

Fig. 4. Impact of $M$ on $\phi(\varsigma)$

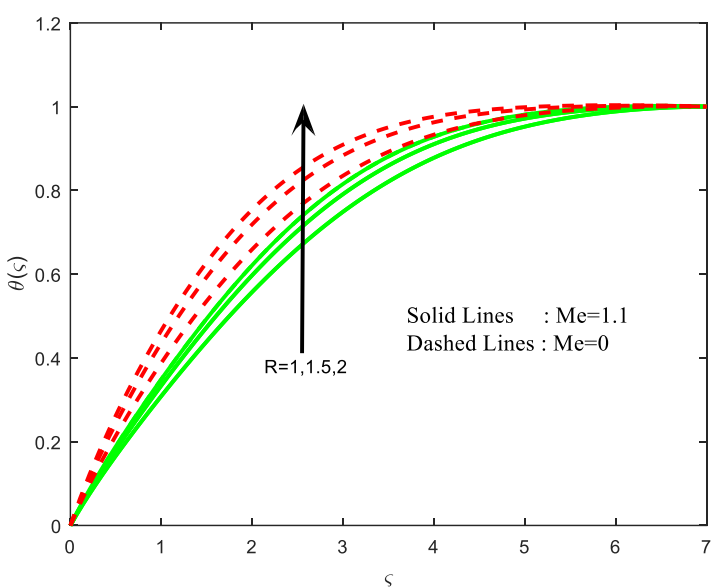

Fig. 6. Impact of $R$ on $\theta(\varsigma)$

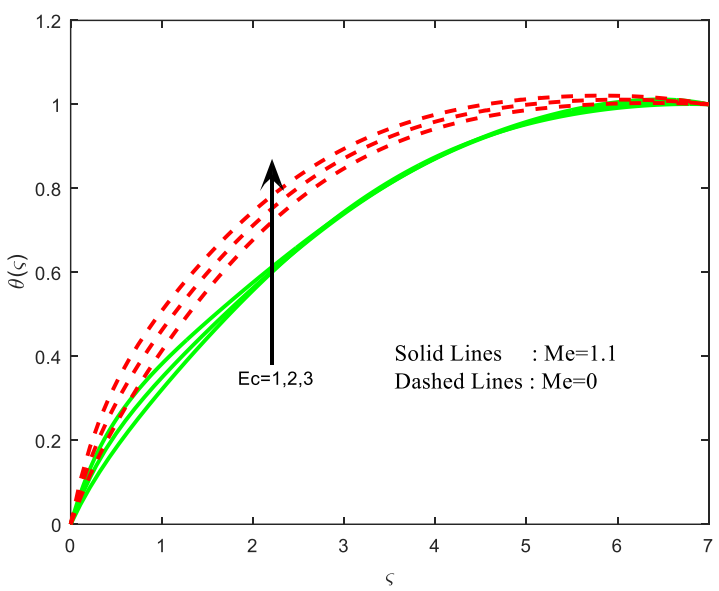

Fig. 8. Impact of $E c$ on $\theta(\varsigma)$

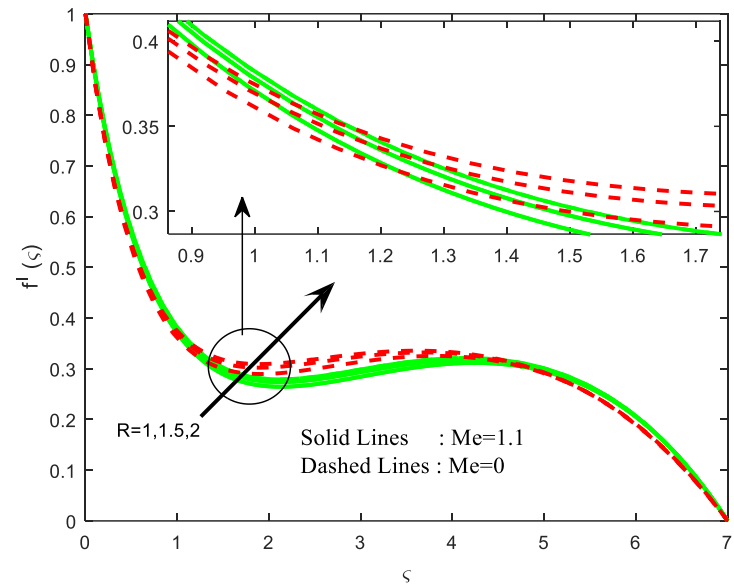

Fig. 5. Impact of $R$ on $f^{\prime}(\varsigma)$

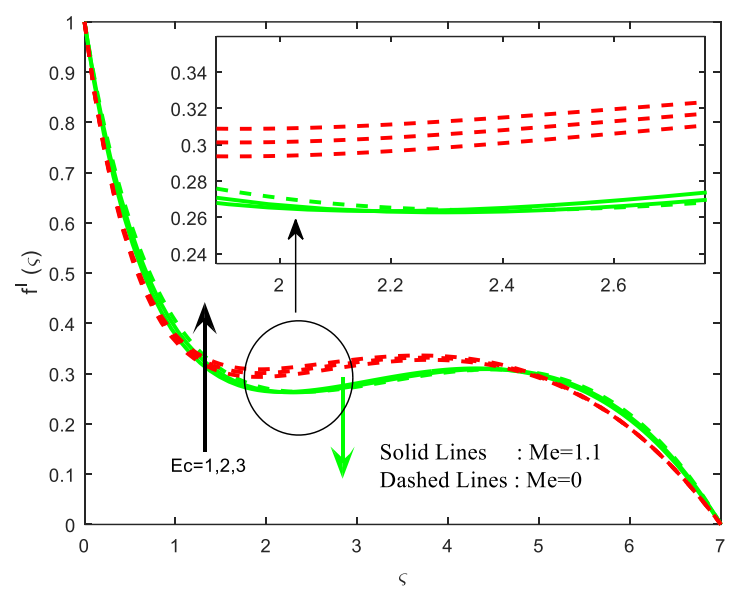

Fig. 7. Impact of $E c$ on $f^{\prime}(\eta)$

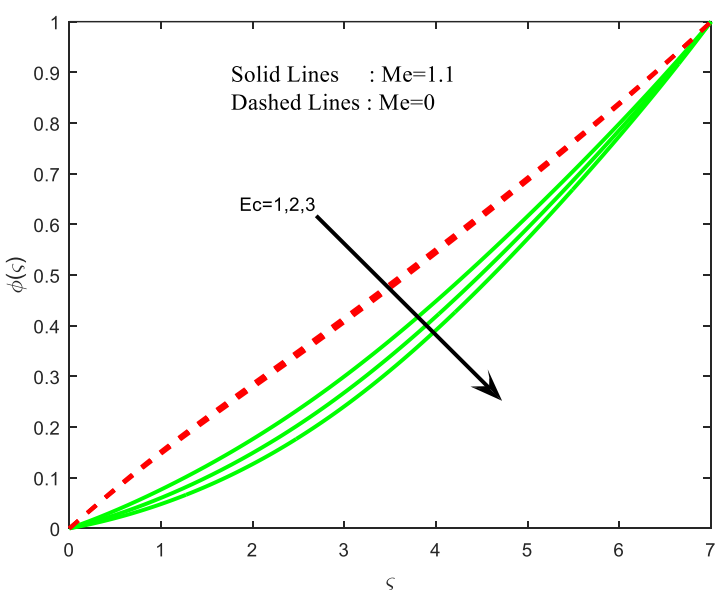

Fig. 9. Impact of $E c$ on $\phi(\varsigma)$ 


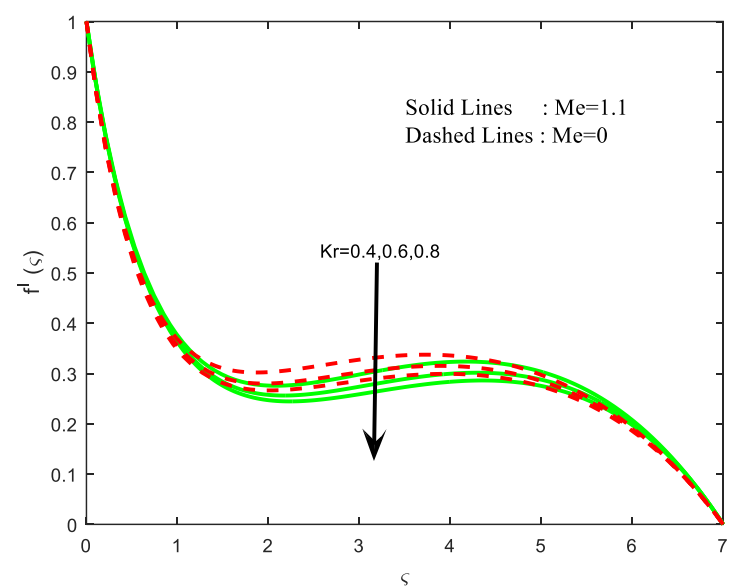

Fig. 10. Impact of $K r$ on $f^{\prime}(\zeta)$

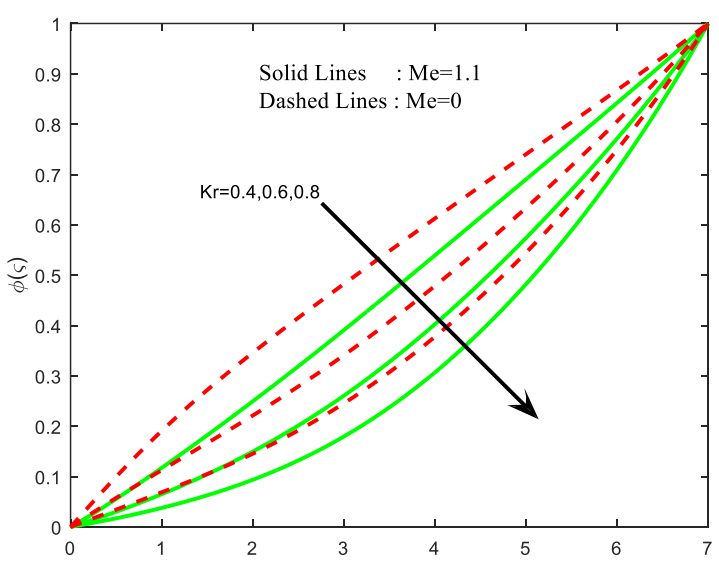

Fig. 12. Impact of $K r$ on $\phi(\eta)$

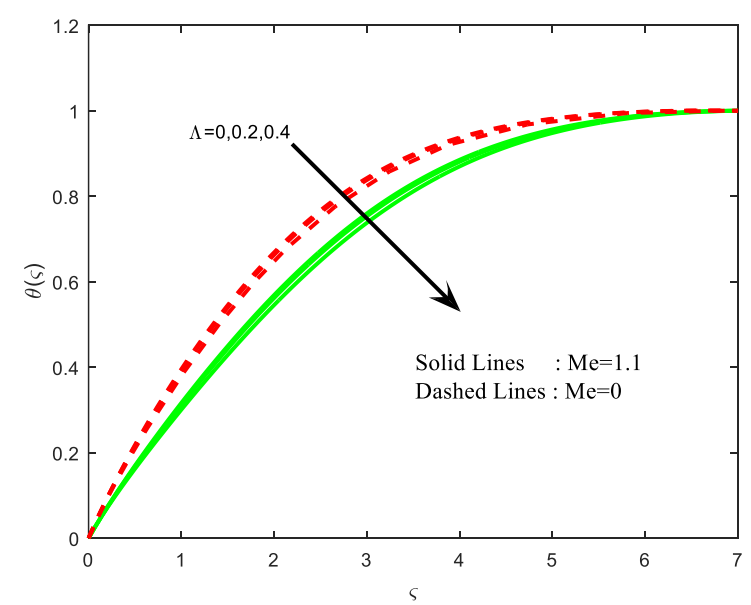

Fig. 14. Impact of $\Lambda$ on $\theta(\eta)$

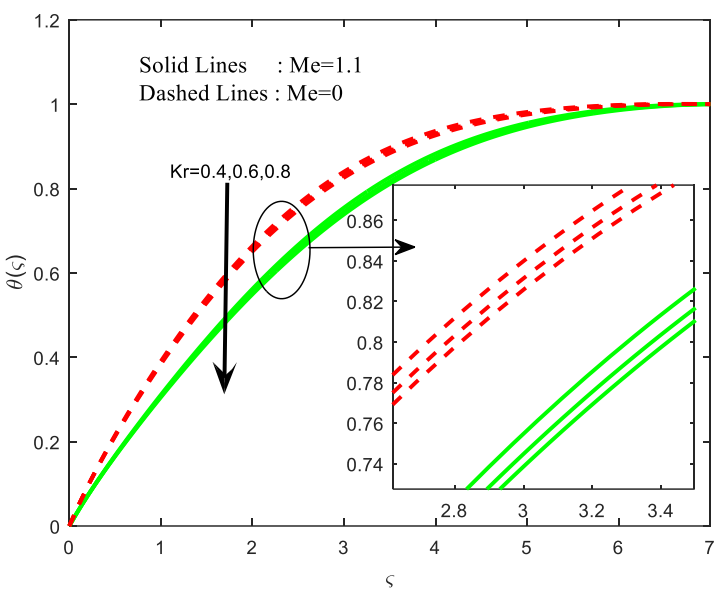

Fig. 11. Impact of $K r$ on $\theta(\zeta)$

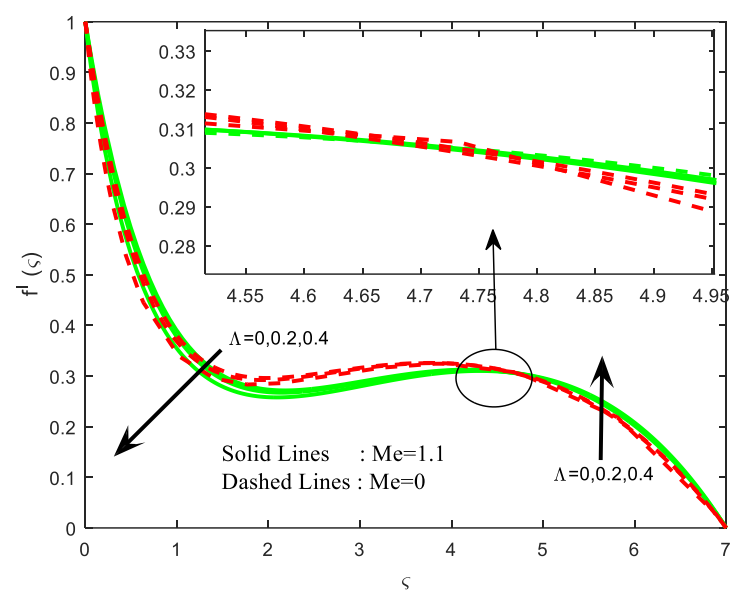

Fig. 13. Impact of $\Lambda$ on $f^{\prime}(\zeta)$

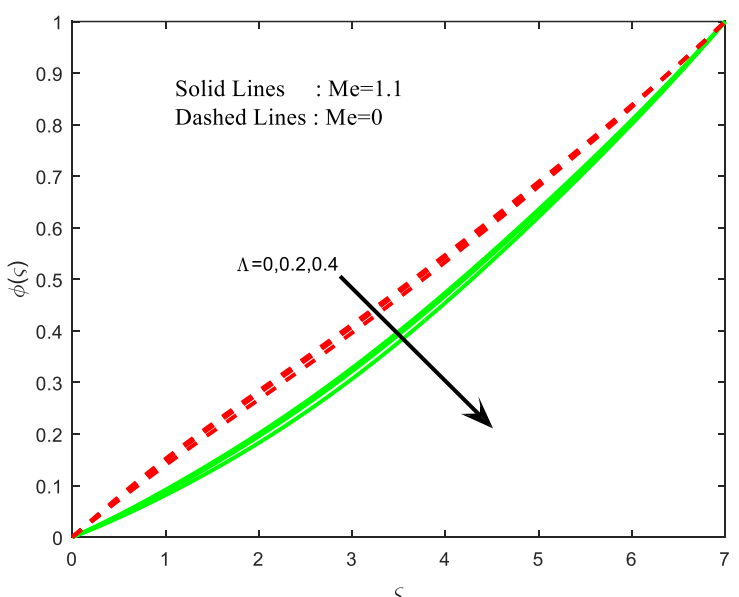

Fig. 15. Impact of $\Lambda$ on $\phi(\zeta)$ 


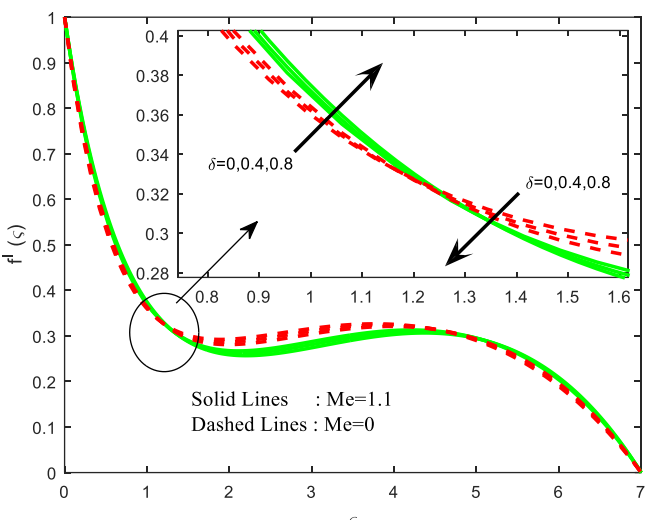

Fig. 16. Impact of $\delta$ on $f^{\prime}(\varsigma)$

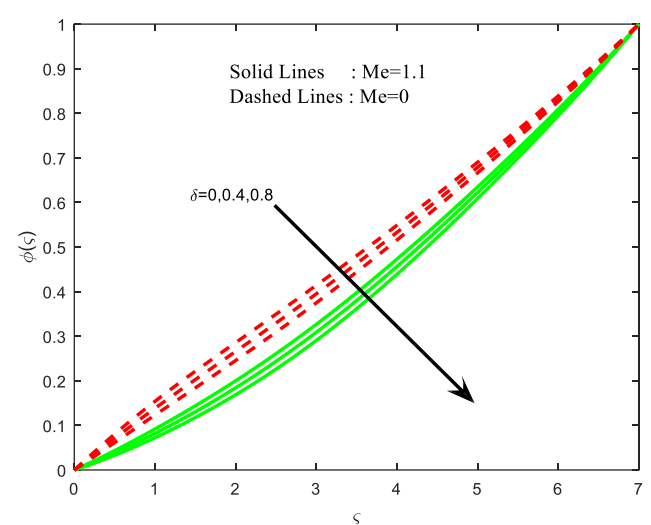

Fig. 18. Impact of $\delta$ on $\phi(\zeta)$

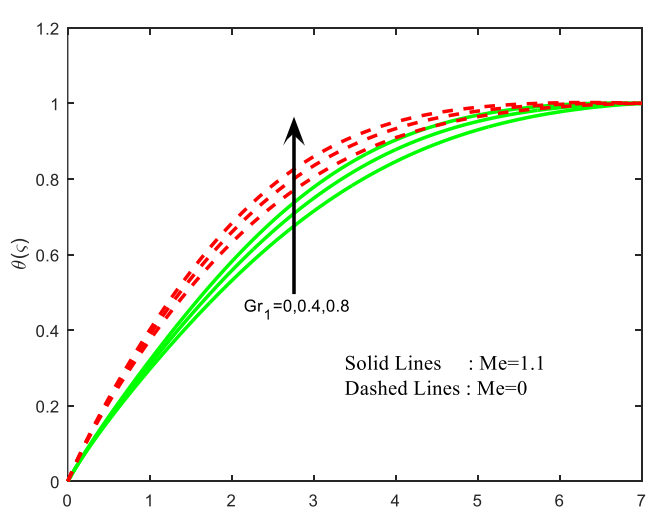

Fig. 20. Impact of $G r_{1}$ on $\theta(\eta)$

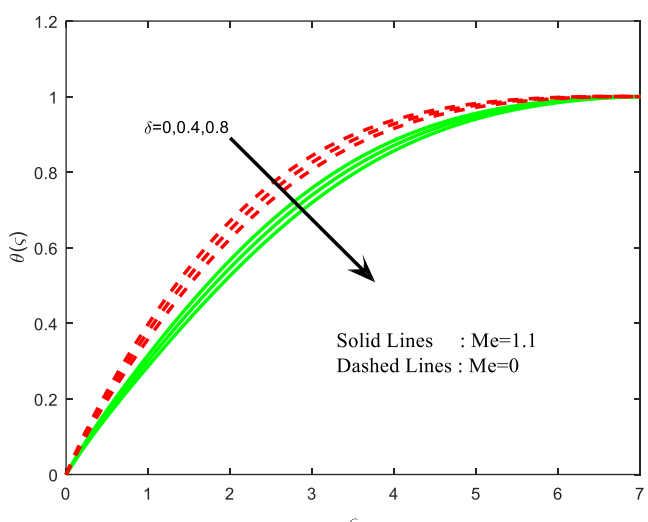

Fig. 17. Impact of $\delta$ on $\theta(\eta)$

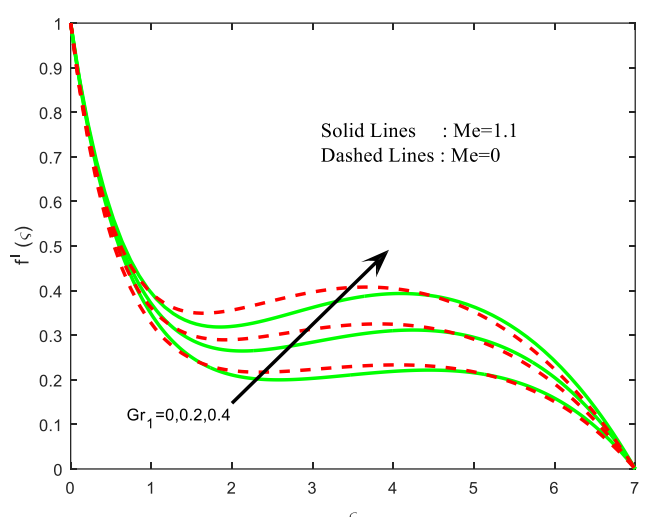

Fig. 19. Impact of $G r_{1}$ on $f^{\prime}(\varsigma)$

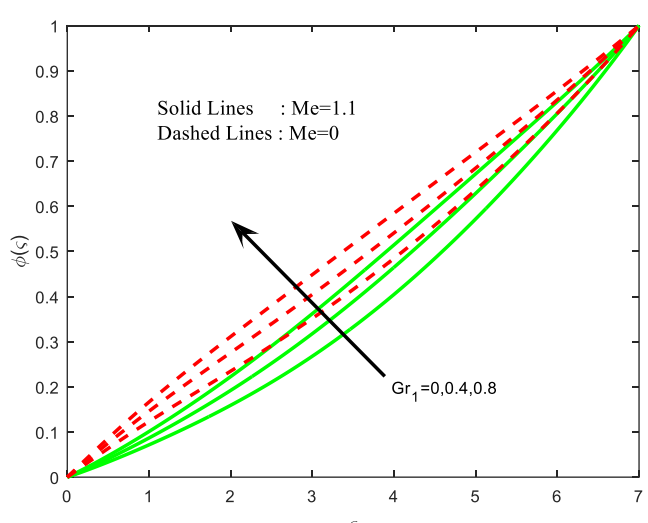

Fig. 21. Impact of $G r_{1}$ on $\phi(\varsigma)$ 


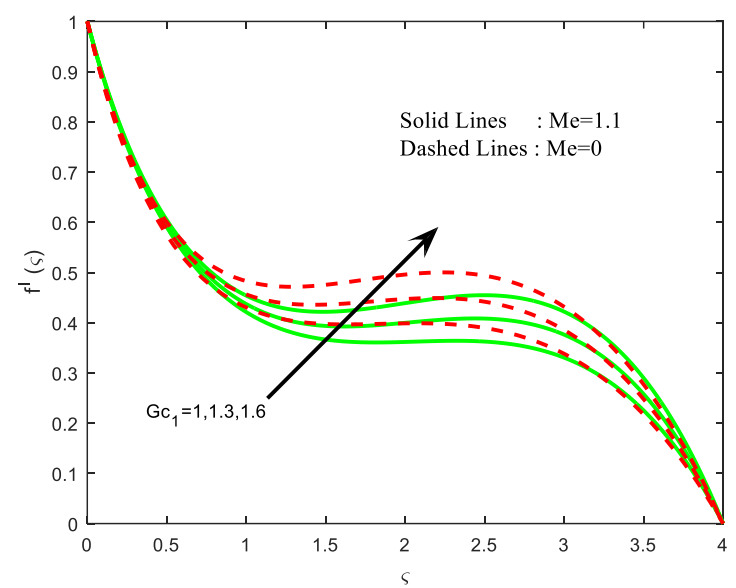

Fig. 22. Impact of $G c_{1}$ on $f^{\prime}(\varsigma)$

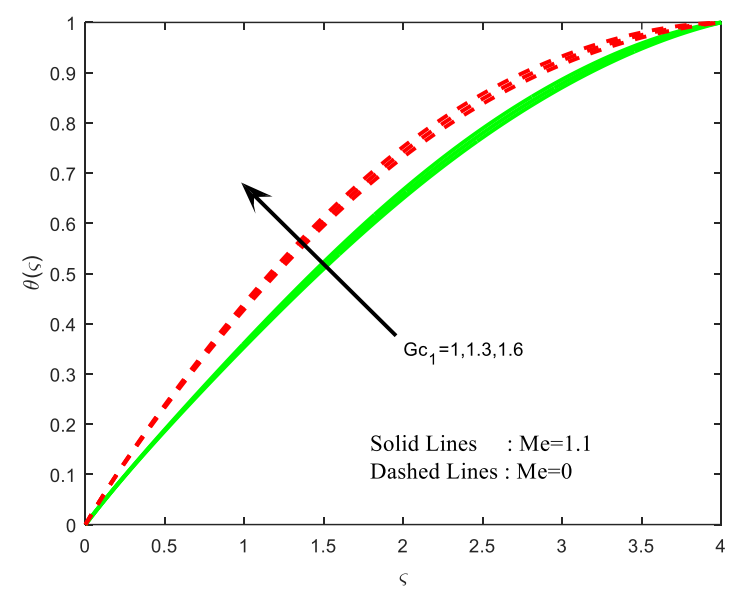

Fig. 23. Impact of $G c_{1}$ on $\theta(\eta)$

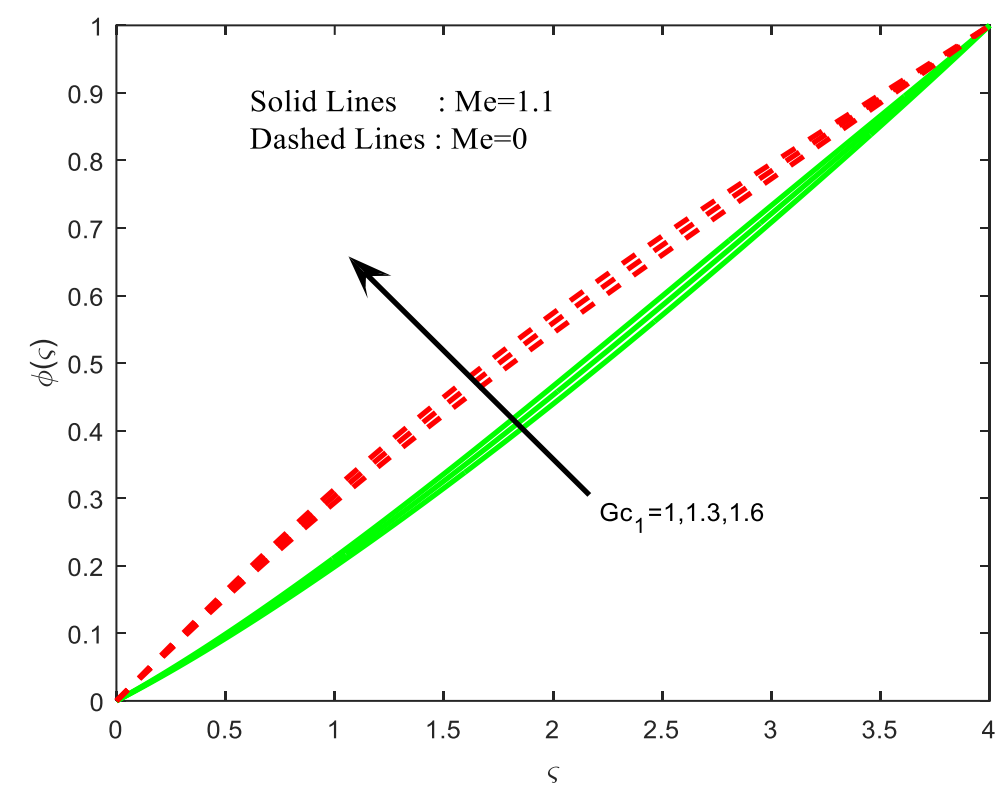

Fig. 24. Impact of $G c_{1}$ on $\phi(\varsigma)$

Table 1 and 2 showed the impact of the relevant parameters on friction factor, local Nusselt number (heat transfer rate) and Sherwood numbers (mass transfer rate) for two cases. From the table 1 , we can state that all the previously mentioned parameters with the exception of $M, K r, \delta$, diminish the heat transfer rate. In the meantime, all the said parameters aside from $G r_{1}, G c_{1}$, improve the mass transfer rate. It is seen that Weissenberg number and wall thickness parameters rise the magnitude of friction factor. Table 2 demonstrates that the parameters are having the exact behaviour as in Table 1. It is worthy to make reference that all quantities are less in case of the absence of melting parameter contrasted with its presence. 


\section{Table 1}

Values of friction factor, Nusselt number and Sherwood number for Diverse Parameters in the presence of melting heat transfer

\begin{tabular}{|c|c|c|c|c|c|c|c|c|c|c|}
\hline$M$ & $R$ & $E c$ & $K r$ & $\Lambda$ & $\delta$ & $G r_{1}$ & $G c_{1}$ & $f^{\prime \prime}(0)$ & $-\theta^{\prime}(0)$ & $-\phi^{\prime}(0)$ \\
\hline 1 & & & & & & & & -1.900469 & -0.551932 & -0.099007 \\
\hline 1.5 & & & & & & & & -2.207576 & -0.551776 & -0.072095 \\
\hline \multirow[t]{22}{*}{2} & & & & & & & & -2.501244 & -0.555224 & -0.054031 \\
\hline & 1 & & & & & & & -1.836544 & -0.552391 & -0.105666 \\
\hline & 1.5 & & & & & & & -1.805724 & -0.602878 & -0.101068 \\
\hline & 2 & & & & & & & -1.787654 & -0.633146 & -0.098330 \\
\hline & & 1 & & & & & & -1.775874 & -0.674736 & -0.089038 \\
\hline & & 2 & & & & & & -1.671252 & -0.896842 & -0.064192 \\
\hline & & 3 & & & & & & -1.584621 & -1.092837 & -0.047314 \\
\hline & & & 0.4 & & & & & -1.828968 & -0.553866 & -0.144891 \\
\hline & & & 0.6 & & & & & -1.842069 & -0.551205 & -0.078024 \\
\hline & & & 0.8 & & & & & -1.849315 & -0.549453 & -0.044033 \\
\hline & & & & 0 & & & & -1.604460 & -0.547565 & -0.112665 \\
\hline & & & & 0.2 & & & & -1.739231 & -0.550337 & -0.108287 \\
\hline & & & & 0.4 & & & & -2.252550 & -0.560047 & -0.098569 \\
\hline & & & & & 0 & & & -1.860016 & -0.568205 & -0.113976 \\
\hline & & & & & 0.4 & & & -1.813333 & -0.536752 & -0.097754 \\
\hline & & & & & 0.8 & & & -1.767675 & -0.506025 & -0.083108 \\
\hline & & & & & & 0 & & -1.869692 & -0.542231 & -0.087389 \\
\hline & & & & & & 0.2 & & -1.836544 & -0.552391 & -0.105666 \\
\hline & & & & & & 0.4 & & -1.803907 & -0.561232 & -0.122778 \\
\hline & & & & & & & 1 & -1.754774 & -0.597266 & -0.238598 \\
\hline & & & & & & & 1.3 & -1.735415 & -0.598973 & -0.246911 \\
\hline & & & & & & & 1.6 & -1.714996 & -0.601037 & -0.255287 \\
\hline
\end{tabular}




\section{Table 2}

Values of friction factor, Nusselt number and Sherwood number for Diverse Parameters in the absence of melting heat transfer

\begin{tabular}{|c|c|c|c|c|c|c|c|c|c|c|}
\hline$M$ & $R$ & $E c$ & $K r$ & $\Lambda$ & $\delta$ & $G r_{1}$ & $G c_{1}$ & $f^{\prime \prime}(0)$ & $-\theta^{\prime}(0)$ & $-\phi^{\prime}(0)$ \\
\hline 1 & & & & & & & & -2.183678 & -0.733625 & -0.208244 \\
\hline 1.5 & & & & & & & & -2.556111 & -0.739621 & -0.167080 \\
\hline \multirow[t]{22}{*}{2} & & & & & & & & -2.924451 & -0.748703 & -0.136975 \\
\hline & 1 & & & & & & & -2.107201 & -0.732795 & -0.217947 \\
\hline & 1.5 & & & & & & & -2.090616 & -0.843194 & -0.223122 \\
\hline & 2 & & & & & & & -2.080351 & -0.916374 & -0.226058 \\
\hline & & 1 & & & & & & -2.098654 & -0.915622 & -0.219796 \\
\hline & & 2 & & & & & & -2.081611 & -1.270611 & -0.223448 \\
\hline & & 3 & & & & & & -2.064682 & -1.611772 & -0.227039 \\
\hline & & & 0.4 & & & & & -2.093522 & -0.734144 & -0.288883 \\
\hline & & & 0.6 & & & & & -2.117411 & -0.731634 & -0.165725 \\
\hline & & & 0.8 & & & & & -2.131092 & -0.729779 & -0.098119 \\
\hline & & & & 0 & & & & -1.769926 & -0.722445 & -0.227774 \\
\hline & & & & 0.2 & & & & -1.957891 & -0.728265 & -0.221676 \\
\hline & & & & 0.4 & & & & -2.065072 & -0.726504 & -0.207535 \\
\hline & & & & & 0 & & & -2.143380 & -0.756335 & -0.235258 \\
\hline & & & & & 0.4 & & & -2.071767 & -0.709568 & -0.201435 \\
\hline & & & & & 0.8 & & & -2.003030 & -0.664083 & -0.170795 \\
\hline & & & & & & 0 & & -2.164174 & -0.721275 & -0.183538 \\
\hline & & & & & & 0.2 & & -2.107201 & -0.732795 & -0.217947 \\
\hline & & & & & & 0.4 & & -2.051755 & -0.742190 & -0.248589 \\
\hline & & & & & & & 1 & -1.987204 & -0.773723 & -0.439687 \\
\hline & & & & & & & 1.3 & -1.945373 & -0.775808 & -0.455278 \\
\hline & & & & & & & 1.6 & -1.902355 & -0.778522 & -0.470711 \\
\hline
\end{tabular}

\section{Conclusions}

The present study addresses the nonlinear convection on Williamson fluid over variable thickness surface with melting transfer parameter. To control the thermal and diffusion boundary, we also included the chemical reaction, thermal radiation and viscous dissipation. The key observations of this investigation can be described as:

- The temperature increases rapidly throughout the boundary layer owing to the release of energy in thermal boundary layer is higher the $M e=0$ case when compared to $M e=1.1$ for increasing values of all governing parameters.

- The wall thickness and Weissenberg number are showed mixed performance on velocity profile due to nonlinear convection in the flow.

- The rate of heat transfer is high in melting surface condition when compared its absence. This result helps us to conclude that, for heating treatment melting surface is useful.

- The time required for the execution is lesser in $M e=0$ case when compared to $M e=1.1$ case in the presence of Eckert number, chemical reaction, nonlinear convection and wall thickness parameters. 


\section{References}

[1] Epstein, M., and D. H. Cho. "Melting heat transfer in steady laminar flow over a flat plate." J. Heat Transfer;(United States) 98, no. 3 (1976). https://doi.org/10.1115/1.3450595

[2] Bachok, Norfifah, Anuar Ishak, and Ioan Pop. "Melting heat transfer in boundary layer stagnation-point flow towards a stretching/shrinking sheet." Physics letters A 374, no. 40 (2010): $4075-4079$. https://doi.org/10.1016/j.physleta.2010.08.032

[3] Ishak, Anuar, Roslinda Nazar, Norfifah Bachok, and loan Pop. "Melting heat transfer in steady laminar flow over a moving surface." Heat and mass transfer 46, no. 4 (2010): 463-468. https://doi.org/10.1007/s00231-010-0592-8

[4] Mustafa, M., T. Hayat, and Awatif A. Hendi. "Influence of Melting Heat Transfer in the Stagnation-Point Flow of a Jeffrey Fluid in the Presence of Viscous Dissipation." Journal of Applied Mechanics 79, no. 2 (2012): 024501. https://doi.org/10.1115/1.4005560

[5] Hayat, Tasawar, Zahid Iqbal, Meraj Mustafa, and Awatif A. Hendi. "Melting heat transfer in the stagnation-point flow of third grade fluid past a stretching sheet with viscous dissipation." Thermal Science 17, no. 3 (2013): 865875. https://doi.org/10.2298/TSCI110405119H

[6] Hayat, T., M. Hussain, M. Awais, and S. Obaidat. "Melting heat transfer in a boundary layer flow of a second grade fluid under Soret and Dufour effects." International Journal of Numerical Methods for Heat \& Fluid Flow 23, no. 7 (2013): 1155-1168. https://doi.org/10.1108/HFF-09-2011-0182

[7] Hayat, Tasawar, Muhammad Farooq, and Ahmad Alsaedi. "Melting heat transfer in the stagnation-point flow of Maxwell fluid with double-diffusive convection." International Journal of Numerical Methods for Heat \& Fluid Flow 24, no. 3 (2014): 760-774. https://doi.org/10.1108/HFF-09-2012-0219

[8] Das, Kalidas, Nilangshu Acharya, and Prabir Kumar Kundu. "Radiative flow of MHD Jeffrey fluid past a stretching sheet with surface slip and melting heat transfer." Alexandria Engineering Journal 54, no. 4 (2015): 815-821. https://doi.org/10.1016/i.aej.2015.06.008

[9] Mabood, Fazle, and Antonio Mastroberardino. "Melting heat transfer on MHD convective flow of a nanofluid over a stretching sheet with viscous dissipation and second order slip." Journal of the Taiwan Institute of Chemical Engineers 57 (2015): 62-68. https://doi.org/10.1016/i.jtice.2015.05.020

[10] Gireesha, B. J., B. Mahanthesh, I. S. Shivakumara, and K. M. Eshwarappa. "Melting heat transfer in boundary layer stagnation-point flow of nanofluid toward a stretching sheet with induced magnetic field." Engineering science and technology, an international journal 19, no. 1 (2016): 313-321. https://doi.org/10.1016/i.jestch.2015.07.012

[11] Mabood, F., and K. Das. "Melting heat transfer on hydromagnetic flow of a nanofluid over a stretching sheet with radiation and second-order slip." The European Physical Journal Plus 131, no. 1 (2016): 1-12. https://doi.org/10.1140/epjp/i2016-16003-1

[12] Krishnamurthy, M. R., B. J. Gireesha, B. C. Prasannakumara, and Rama Subba Reddy Gorla. "Thermal radiation and chemical reaction effects on boundary layer slip flow and melting heat transfer of nanofluid induced by a nonlinear stretching sheet." Nonlinear Engineering 5, no. 3 (2016): 147-159. https://doi.org/10.1515/nleng-2016-0013

[13] Animasaun, Isaac Lare. "Melting heat and mass transfer in stagnation point micropolar fluid flow of temperature dependent fluid viscosity and thermal conductivity at constant vortex viscosity." Journal of the Egyptian Mathematical Society 25, no. 1 (2017): 79-85. https://doi.org/10.1016/j.joems.2016.06.007

[14] Khan, Masood, and Ali Saleh Alshomrani. "Characteristics of melting heat transfer during flow of Carreau fluid induced by a stretching cylinder." The European Physical Journal E 40, no. 1 (2017): 1-9. https://doi.org/10.1140/epje/i2017-11495-6

[15] Sheikholeslami, Mohsen, and Houman B. Rokni. "Melting heat transfer influence on nanofluid flow inside a cavity in existence of magnetic field." International Journal of Heat and Mass Transfer 114 (2017): 517-526. https://doi.org/10.1016/j.ijheatmasstransfer.2017.06.092

[16] Sheikholeslami, M., M. Jafaryar, K. Bateni, and D. D. Ganji. "Two phase modeling of nanofluid flow in existence of melting heat transfer by means of HAM." Indian Journal of Physics 92, no. 2 (2018): $205-214$. https://doi.org/10.1007/s12648-017-1090-3

[17] Viskanta, R., and R. J. Grosh. "Boundary layer in thermal radiation absorbing and emitting media." International Journal of Heat and Mass Transfer 5, no. 9 (1962): 795-806. https://doi.org/10.1016/0017-9310(62)90180-1

[18] Gupta, P. S., and A. S. Gupta. "Radiation effect on hydromagnetic convection in a vertical channel." International Journal of Heat and Mass Transfer 17, no. 12 (1974): 1437-1442. https://doi.org/10.1016/0017-9310(74)90053-2

[19] Lavanya, Bommanna. "MHD Rotating Flow Through a Porous Medium with Heat and Mass Transfer." Journal of Advanced Research in Fluid Mechanics and Thermal Sciences 54, no. 2 (2019): 221-231.

[20] Ali, Mir Mosaddeq, T. S. Chen, and Bassem F. Armaly. "Natural convection-radiation interaction in boundary-layer flow overhorizontal surfaces." AIAA journal 22, no. 12 (1984): 1797-1803. https://doi.org/10.2514/3.8854 
[21] Hossain, M. A., and H. S. Takhar. "Radiation effect on mixed convection along a vertical plate with uniform surface temperature." Heat and Mass transfer 31, no. 4 (1996): 243-248. https://doi.org/10.1007/BF02328616

[22] Israel-Cookey, C., A. Ogulu, and V. B. Omubo-Pepple. "Influence of viscous dissipation and radiation on unsteady MHD free-convection flow past an infinite heated vertical plate in a porous medium with time-dependent suction." International Journal of Heat and Mass Transfer 46, no. 13 (2003): 2305-2311. https://doi.org/10.1016/S0017-9310(02)00544-6

[23] Raptis, Andreas, Christos Perdikis, and Harmindar S. Takhar. "Effect of thermal radiation on MHD flow." Applied Mathematics and computation 153, no. 3 (2004): 645-649. https://doi.org/10.1016/S0096-3003(03)00657-X

[24] Cortell, Rafael. "Effects of viscous dissipation and radiation on the thermal boundary layer over a nonlinearly stretching sheet." Physics Letters A 372, no. 5 (2008): 631-636. https://doi.org/10.1016/i.physleta.2007.08.005

[25] Mohamed, R. A., and S. M. Abo-Dahab. "Influence of chemical reaction and thermal radiation on the heat and mass transfer in MHD micropolar flow over a vertical moving porous plate in a porous medium with heat generation." International Journal of Thermal Sciences 48, no. 9 (2009): $1800-1813$. https://doi.org/10.1016/i.ijthermalsci.2009.01.019

[26] Mukhopadhyay, S. "Effect of thermal radiation on unsteady mixed convection flow and heat transfer over a porous stretching surface in porous medium." International journal of heat and mass transfer 52, no. 13-14 (2009): 32613265. https://doi.org/10.1016/i.ijheatmasstransfer.2008.12.029

[27] Nagasasikala, Madduleti, and Bommanna Lavanya. "Heat and mass transfer of a MHD flow of a nanofluid through a porous medium in an annular, circular region with outer cylinder maintained at constant heat flux." CFD Letters 11, no. 9 (2019): 32-58.

[28] Bhattacharyya, Krishnendu, and G. C. Layek. "Effects of suction/blowing on steady boundary layer stagnation-point flow and heat transfer towards a shrinking sheet with thermal radiation." International Journal of Heat and Mass Transfer 54, no. 1-3 (2011): 302-307. https://doi.org/10.1016/i.ijheatmasstransfer.2010.09.043

[29] Pramanik, S. "Casson fluid flow and heat transfer past an exponentially porous stretching surface in presence of thermal radiation." Ain Shams Engineering Journal 5, no. $1 \quad$ (2014): $205-212$. https://doi.org/10.1016/i.asej.2013.05.003

[30] Jonnadula, Manjula, Padma Polarapu, and Gnaneswara Reddy. "Influence of thermal radiation and chemical reaction on MHD flow, heat and mass transfer over a stretching surface." Procedia Engineering 127 (2015): 13151322. https://doi.org/10.1016/i.proeng.2015.11.489

[31] Aman, Sidra, Ilyas Khan, Zulkhibri Ismail, and Mohd Zuki Salleh. "Impacts of gold nanoparticles on MHD mixed convection Poiseuille flow of nanofluid passing through a porous medium in the presence of thermal radiation, thermal diffusion and chemical reaction." Neural Computing and Applications 30, no. 3 (2018): $789-797$. https://doi.org/10.1007/s00521-016-2688-7

[32] Bhatti, Muhammad Mubashir, and Mohammad Mehdi Rashidi. "Effects of thermo-diffusion and thermal radiation on Williamson nanofluid over a porous shrinking/stretching sheet." Journal of Molecular Liquids 221 (2016): 567573. https://doi.org/10.1016/j.molliq.2016.05.049

[33] Makinde, O. D., and I. L. Animasaun. "Bioconvection in MHD nanofluid flow with nonlinear thermal radiation and quartic autocatalysis chemical reaction past an upper surface of a paraboloid of revolution." International Journal of Thermal Sciences 109 (2016): 159-171. https://doi.org/10.1016/j.ijthermalsci.2016.06.003

[34] Pandey, Alok Kumar, and Manoj Kumar. "Natural convection and thermal radiation influence on nanofluid flow over a stretching cylinder in a porous medium with viscous dissipation." Alexandria Engineering Journal 56, no. 1 (2017): 55-62. https://doi.org/10.1016/i.aej.2016.08.035

[35] Hayat, T., Gulnaz Bashir, M. Waqas, and A. Alsaedi. "MHD 2D flow of Williamson nanofluid over a nonlinear variable thicked surface with melting heat transfer." Journal of Molecular Liquids 223 (2016): 836-844. https://doi.org/10.1016/i.molliq.2016.08.104

[36] Nadeem, S., S. T. Hussain, and Changhoon Lee. "Flow of a Williamson fluid over a stretching sheet." Brazilian journal of chemical engineering 30, no. 3 (2013): 619-625. https://doi.org/10.1590/S0104-66322013000300019

[37] Saravana, R., S. Sreekanth, S. Sreenadh, and R. Hemadri Reddy. "Mass transfer effects on MHD viscous flow past an impulsively started infinite vertical plate with constant mass flux." Advances in Applied Science Research 2, no. 1 (2011): 221-229. 